\title{
Kinetic energy driven superconductivity and superfluidity
}

\author{
J. E. Hirsch \\ Department of Physics, University of California, San Diego \\ La Jolla, CA 92093-0319
}

\begin{abstract}
The theory of hole superconductivity proposes that superconductivity is driven by lowering of quantum kinetic energy and is associated with expansion of electronic orbits and expulsion of negative charge from the interior to the surface of superconductors and beyond. This physics provides a dynamical explanation of the Meissner effect. Here we propose that similar physics takes place in superfluid helium 4. Experimental manifestations of this physics in ${ }^{4} \mathrm{He}$ are the negative thermal expansion of ${ }^{4} \mathrm{He}$ below the $\lambda$ point and the "Onnes effect", the fact that superfluid helium will creep up the walls of the container and escape to the exterior. The Onnes effect and the Meissner effect are proposed to originate in macroscopic zero point rotational motion of the superfluids. It is proposed that this physics indicates a fundamental inadequacy of conventional quantum mechanics.
\end{abstract}

PACS numbers:

\section{INTRODUCTION}

There exist remarkable similarities in many aspects of the physical behavior exhibited by superfluid ${ }^{4} \mathrm{He}$ and superconductors. This has been repeatedly found experimentally as well as theoretically and discussed, particularly by F. London and K. Mendelssohn [1-3], in the early days of research in these fields. In this paper we point out that some qualitatively new physics recently predicted to exist in superconductors within the theory of hole superconductivity also appears to have a remarkable counterpart in superfluid ${ }^{4} \mathrm{He}$.

The theory of hole superconductivity differs in fundamental ways from conventional BCS-London theory. It proposes [4] that superconductors expel negative charge from the interior to the surface, a process that is associated with expansion of the electronic wavefunctions and driven by lowering of the quantum kinetic energy. In the region of excess negative charge that results within a

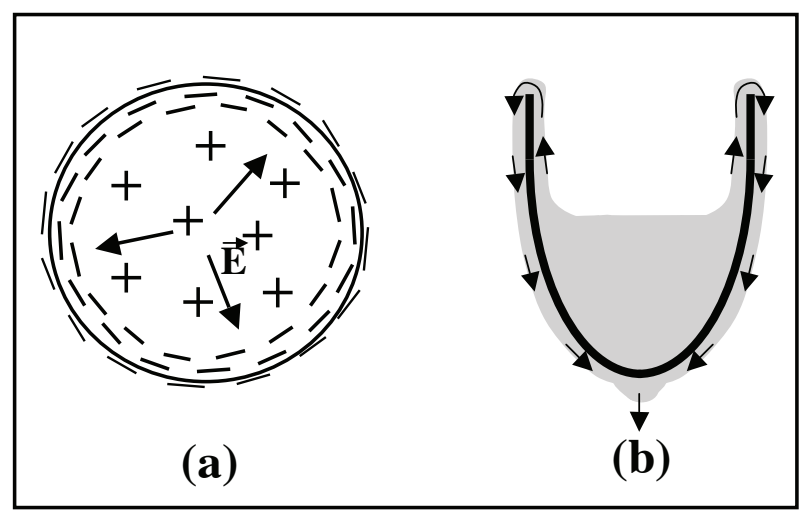

FIG. 1: (a) The superconductor expels negative charge from the interior to the surface. A small amount of charge spills out beyond the surface. (b) Superfluid ${ }^{4} \mathrm{He}$ continuously expels mass from the bulk, that creeps up the inner surface of the container and creeps down the outer surface, at a uniform rate.
London penetration depth of the surface a macroscopic spin current is predicted to flow in the absence of applied fields, a kind of zero-point motion of the superfluid[5]. Furthermore the expelled negative charge is predicted to 'seep out' from the surface of the body to the exterior [6] (see Fig. 1(a)).

An equivalent physics in superfluid He would correspond to a tendency of the superfluid to expel mass. And indeed such an effect exists, the 'Onnes effect' associated with 'Rollin films', shown schematically in Fig. 1(b). Superfluid He will spontaneously creep up the walls of the container, defying gravity, and escape to the exterior. This effect, first encountered (but not understood) by Onnes in 1922 [7] and investigated many years later by Rollin [8] and especially by Daunt and Mendelssohn[9] in a series of detailed experiments, is arguably the most remarkable property of superfluid He. In this paper we propose that the essential physics behind the phenomena displayed in Fig. 1(a) and Fig. 1(b) for superconductors and superfluids respectively is the same. One difference however is that in the superfluid the mass continuously flows out, while in the superconductor the expelled negative charge is held back by the strong electric force that results from the macroscopic charge inhomogeneity.

The current understanding of ${ }^{4} \mathrm{He}$ is principally based on the work of Feynman as described in Ref. [10]. Unfortunately, at the outset of that review paper Feynman states 'We shall omit references to the phenomena involved in the Rollin film'. Thus we have to focus our attention on earlier theoretical and experimental work that properly focused on what we believe is the key physics of superfluid ${ }^{4} \mathrm{He}$.

Mendelssohn [11, 12] and F. London [13] have pointed out the remarkable similarity in the 'transfer phenomena' exhibited by superconductors and superfluids, namely the flow of mass in superfluid He films depicted in Fig. 1(b) as well as through capillaries, and the flow of charge within the London penetration depth of the surface that occurs in a superconducting wire carrying a current. They have proposed that the speed at which these 'transfer phenomena' occur is given by a 'quantum condition' 
involving the fundamental constant $\hbar$. For ${ }^{4} H e$, a similar proposal had been made earlier by Bilj, de Boer and Michels 14]. These proposals are not regarded as valid in the contemporary view of ${ }^{4} \mathrm{He}$ [15-18], having been superseded by theories that ascribe the film formation and flow in ${ }^{4} \mathrm{He}$ exclusively to Van der Waals forces. In this paper we argue that the original proposals of Mendelssohn, Bilj et al and F. London were indeed correct, which implies that fundamental physics of ${ }^{4} \mathrm{He}$ is missed in the currently accepted understanding of this system.

In addition, being macroscopic quantum systems, superfluids and superconductors provide us with a 'window' through which we can peer into the microscopic world of quantum mechanics and understand it in a new way. In the last sections of this paper we discuss the implications of this physics of superfluids and superconductors to the understanding of fundamental quantum mechanics.

\section{KINETIC ENERGY DRIVEN SUPERCONDUCTIVITY}

Within conventional BCS theory [19], carriers lower their potential energy and increase their kinetic energy in the transition to superconductivity. Just the opposite is predicted to occur within the theory of hole superconductivity [4]. Experimental evidence for kinetic energy lowering in the transition to superconductivity in cuprates and pnictides has been seen in optical properties [21].

The theory predicts that as the system goes superconducting an expansion of the electronic wavefunction occurs, driven by kinetic energy lowering, from linear extension $k_{F}^{-1}\left(k_{F}=\right.$ Fermi wavevector) in the normal state, to $2 \lambda_{L}$ in the superconducting state, with $\lambda_{L}$ the London penetration depth $[5,[20]$. The normal state can be viewed as electrons residing in non-overlapping 'orbits' of radius $k_{F}^{-1}$, which is of order of the interatomic distances since the band is almost full (hole conduction is required). The normal state magnetic susceptibility is given by the Larmor susceptibility with orbit radius $k_{F}^{-1}$ :

$$
\chi_{\text {Larmor }}\left(r=k_{F}^{-1}\right)=-\frac{n e^{2}}{4 m_{e} c^{2}} k_{F}^{-2}=-\frac{1}{3} \mu_{B}^{2} g\left(\epsilon_{F}\right)
$$

with $n$ the number of electrons per unit volume, $g\left(\epsilon_{F}\right)=$ $3 n / 2 \epsilon_{F}$ the density of states at the Fermi energy and $\mu_{B}=|e| \hbar / 2 m_{e} c$ the Bohr magneton, yielding Landau diamagnetism. Because the orbits are non-overlapping, each electron can be at any position in its orbit relative to other electrons; there is no 'phase coherence'. As the system goes superconducting, the orbits expand to mesoscopic radius $2 \lambda_{L}$ and the susceptibility is given by

$$
\chi_{\text {Larmor }}\left(r=2 \lambda_{L}\right)=-\frac{n e^{2}}{4 m_{e} c^{2}}\left(2 \lambda_{L}\right)^{2}=-\frac{1}{4 \pi}
$$

(using that $1 / \lambda_{L}^{2}=4 \pi n e^{2} / m_{e} c^{2}$ ), indicating perfect diamagnetism. Because the orbit radius is now much larger

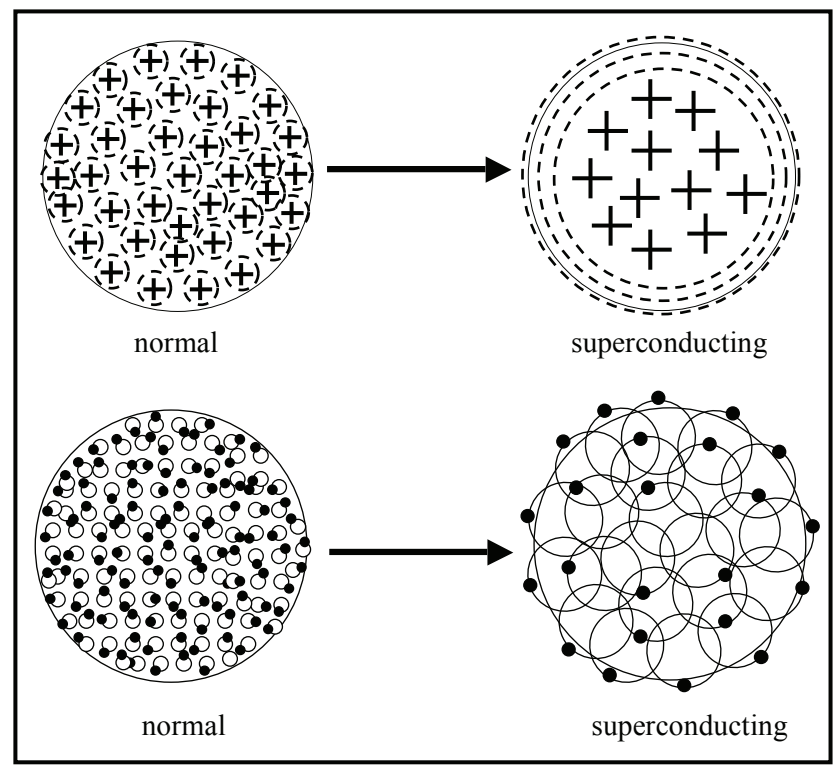

FIG. 2: As electrons expand their orbits from radius $k_{F}^{-1}$ (left lower panel) to radius $2 \lambda_{L}$ (right lower panel), negative charge moves from the interior to the surface (upper panel). The 'phase' of each orbit (defined as the position of the electron in its orbit, denoted by the black dot) is random in the normal state because the orbits are non-overlapping. When the orbits overlap in the superconducting state, the phase of one orbit is correlated to the phase of all other orbits and there is 'macroscopic phase coherence' to avoid collisions.

than the interelectronic distances the orbits are highly overlapping and long-range phase coherence is required to avoid collisions that would raise the energy of the system. This orbit expansion has associated with it negative charge expulsion from the interior to the surface of superconductors, as depicted schematically in Fig. 2.

In the $2 \lambda_{L}$ orbits, opposite spin electrons traverse their orbits in opposite directions. The electrons acquire their orbital speed through the spin-orbit interaction of the magnetic moment of the electron with the ionic charge background as the orbits expand from the microscopic scale $\left(k_{F}^{-1}\right)\left(k_{F}=\right.$ Fermi wavevector $)$ to $2 \lambda_{L}$, through a 'quantum spin Hall effect' [5]. The speed of the electrons is derived from the strength of the spin orbit interaction as given by Dirac's equation with the electric field generated by the ionic background and yields [5, 22 ]

$$
\mathbf{v}_{\sigma}^{0}=-\frac{\hbar}{4 m_{e} \lambda_{L}} \hat{\sigma} \times \hat{n}
$$

where $\hat{n}$ is the outward normal to the surface. The orbital angular momentum of electrons in the $2 \lambda_{L}$ orbits is

$$
L=m_{e} v_{\sigma}^{0}\left(2 \lambda_{L}\right)=\frac{\hbar}{2}
$$

The superposition of these orbital motions gives rise to a macroscopic spin current flowing within a London penetration depth of the surface of superconductors, parallel 
to the surface 23]. This is a macroscopic zero point motion of the superconductor which is predicted to exist even at zero temperature [5].

From Heisenberg's uncertainty principle one infers that a particle confined to a linear distance $\Delta x$ undergoes zero-point motion with minimum momentum $p=\hbar /(2 \Delta x)$ and speed $v=\hbar /(2 m \Delta x)$, with $m$ the mass. Therefore we can interpret the spin current speed Eq. (3) as zero-point motion originating in the "confinement' of the carriers in a region of width $\Delta x=2 \lambda_{L}$ from the surface. It is important to note however that the net speed of the superfluid spin current is parallel to the surface rather than in the 'confinement' direction which is perpendicular to the surface.

In the presence of an external magnetic field, electrons in the expanding orbits acquire an additional speed due to the action of the Lorentz force, giving rise to a surface current that will screen the magnetic field. Thus, this physics provides a dynamical explanation of the Meissner effect [24]. As discussed in Ref. [5], the speed of an electron near the surface in the presence of an applied magnetic field is

$$
v_{\sigma}=v_{\sigma}^{0} \pm \frac{e}{m_{e} c} B \lambda_{L}
$$

so that it increases (decreases) for spin antiparallel (parallel) to the magnetic field. Superconductivity is destroyed when the speed of the electrons with spin parallel to the magnetic field goes to zero, at the critical field given by [5]

$$
B_{s}=\frac{\hbar c}{4|e| \lambda_{L}^{2}} .
$$

Therefore, electrons carrying the charge current in the presence of a magnetic field have maximum speed $2 v_{\sigma}^{0}$. The expression Eq. (6) is essentially $H_{c 1}$, the lower critical field of type II superconductors [19], and the charge current corresponding to half the carriers moving at speed $2 v_{\sigma}^{0}$ (or all the carriers moving at speed $v_{\sigma}^{0}$ in the same direction) gives the critical current according to Silsbee's criterion [19]:

$$
J_{c}=n_{s} e v_{\sigma}^{0}=\frac{c}{4 \pi \lambda_{L}} H_{c 1}
$$

using the well-known expression [19]

$$
\frac{1}{\lambda_{L}^{2}}=\frac{4 \pi n_{s} e^{2}}{m_{e} c^{2}}
$$

with $n_{s}$ the superfluid density. The excess negative charge near the surface is $\rho_{-}=e n_{s} v_{\sigma}^{0} / c$, and the critical current Eq. (7) can be understood as arising from $\rho_{-}$propagating at the speed of light [23].

\section{KINETIC ENERGY DRIVEN SUPERFLUIDITY}

The fact that kinetic energy lowering plays a dominant role in the physics of liquid helium has been known for a long time 25.

As discussed by London 26], the average interatomic distance in liquid helium $(4.0 \AA)$ is considerably larger than what would correspond to the minimum in the Van der Waals potential energy $(3.0 \AA)$. The molar volume of ${ }^{4} \mathrm{He}$ at zero pressure is almost three times as large as the volume for which a closed-pack arrangement of $\mathrm{He}$ atoms has minimum potential energy. The reason that He at low temperatures does not optimize its potential energy is of course that the zero-point kinetic energy at the distance of minimum potential energy would be way too high [26]. If packed at that density, the system will expand from this classically optimal configuration, driven by quantum kinetic energy lowering, to the actual density that minimizes the sum of potential and kinetic energies. And quantum zero-point motion is of course the reason why helium does not solidify but remains liquid under its own vapor pressure even at zero temperature, unlike any other substance 25].

In addition, when cooled below the $\lambda$ point the system expands further: the thermal expansion coefficient of helium changes sign from positive above the $\lambda$ point to negative below [27]. Unlike the case of water crystallizing into ice, where the expansion can be understood from classical geometrical and potential energy considerations, there is no classical explanation for this behavior for the structureless He atoms. The Hamiltonian for ${ }^{4} \mathrm{He}$ is given by

$$
H=-\sum_{i} \frac{\hbar^{2}}{2 m_{H e}} \nabla_{i}^{2}+\frac{1}{2} \sum_{i \neq j} U\left(\left|\vec{r}_{i}-\vec{r}_{j}\right|\right) \equiv K+U
$$

and the energy of the system is the expectation value

$$
E=<\Psi|K| \Psi>+<\Psi|U| \Psi>
$$

with the many-body wavefunction $\mid \Psi>$. In the normal liquid, the expectation value $\langle\Psi|U| \Psi\rangle$ is already larger than what it would be for a higher density, since the average interatomic distance is a factor 1.3 larger than what would correspond to the minimum in the Van der Waals potential energy between atoms. When the system is cooled below the $\lambda$ point the average interatomic distance increases further, by about 5 parts in 1,000 between $T_{\lambda}$ and $T=0$. This implies that the average potential energy $<\Psi|U| \Psi>$ increases further, hence the average kinetic energy $<\Psi|K| \Psi>$ has to decrease. Thus, the fact that ${ }^{4} \mathrm{He}$ expands rather than contracts as the temperature is lowered below the $\lambda$ point is clearly driven by kinetic energy lowering, i.e. 'quantum pressure'. This (experimentally verified) expansion of the system as it becomes superfluid parallels the (not yet experimentally verified) orbit expansion and charge expulsion that we predict occurs when a metal enters the superconducting state, also driven by kinetic energy lowering [4, 28].

However, the kinetic energy lowering is much larger than what would result from this volume expansion. In the normal state of ${ }^{4} \mathrm{He}$ the atomic wavefunction is confined to a "cage" determined by its neighboring 
atoms [29], with zero-point energy $\sim h^{2} / 8 m \delta^{2}$, with $\delta$ the average interatomic distance. An increase in $\delta$ of only 5 parts in 1,000 cannot of course account for the condensation energy which is of order $k_{B} T_{\lambda}\left(T_{\lambda}=2.19^{\circ} \mathrm{K}\right)$, given that $h^{2} / 8 m \delta^{2} k_{B}=3.71^{\circ} K$ for interatomic distance $\delta \sim 4 \AA$. Consequently, in addition to $\delta$ expanding the atomic wavefunctions must expand way beyond $\delta$ and strongly overlap with each other, as depicted in the lower right panel of Fig. 2 for superconductors, thus lowering the quantum kinetic energy by an amount of order $k_{B} T_{\lambda}$. For superconductors, the transition is associated with expansion of the electron wavefunction from $k_{F}^{-1}$, the interelectronic distance, to $2 \lambda_{L}$. There is no analog of the $\lambda_{L}$ length for ${ }^{4} \mathrm{He}$, so it is reasonable to conclude that for ${ }^{4} \mathrm{He}$ the wavelength expands from $\sim \delta$ to the entire region allowed by geometrical constraints.

Incidentally, negative thermal expansion has also been proposed to be associated with kinetic energy lowering in metallic ferromagnets [30].

\section{THICKNESS OF ${ }^{4} \mathrm{He}$ FILMS}

The films that ${ }^{4} \mathrm{He}$ forms below the $\lambda$ point on a vertical surface dipped in the fluid are found to be remarkably thick, typically of order $300 \AA^{\circ}$ or 80 atomic layers at a height $\sim 1 \mathrm{~cm}$ above the surface. In contrast, films at temperatures above the $\lambda$ point are found to be substantially thinner, sometimes as thin as $10 \AA[15-18]$.

This observation suggest that the thickness of films is intimately tied to the superfluid character. Remarkably however, the generally accepted view is that the film thickness is determined solely by Van der Waals forces between the ${ }^{4} \mathrm{He}$ atoms and the surface. In a simple description, the equation relating the film thickness $d$ to the height above the surface $z$ is taken to be 15 ]

$$
m_{H e} g z-\frac{m_{H e} \alpha}{d^{3}}=0
$$

with $g$ the acceleration of gravity and $\alpha$ a constant describing the potential energy of interaction of $\mathrm{He}$ atoms and the surface, originating in Van der Waals forces. This view implies that the film thickness should be the same below and above the $\lambda$ point, the observation that it is not is attributed to the different heat conduction properties of $\mathrm{He}$ below and above the $\lambda$ point $15-18$.

Instead, the unified physical picture proposed in this paper suggests that the driving mechanism for the formation of the thick films below the $\lambda$ point is kinetic energy lowering. This mechanism of film formation was in fact proposed long ago by Bilj et al[14], but is not considered valid in the contemporary view. The equation relating height $z$ and thickness $d$ is 14 ]

$$
m_{H e} g z-\frac{h^{2}}{8 m_{H e} d^{2}}=0 .
$$

The second term originates in the fact that as an atom is added to the film it pays zero-point kinetic energy $h^{2} / 8 m_{H e} d^{2}$, however the zero-point energy of all the atoms at that height is lowered by twice that amount due to the slight increase in $d$ in adding the extra atom.

Equations (11) and (12) make qualitatively different predictions on the power-law dependence of film thickness versus height, namely $d \propto z^{-1 / 3}$ and $d \propto z^{-1 / 2}$ respectively. Experiments have not clearly favored one over the other $15-18]$. The magnitude of the observed thicknessess is approximately consistent with either explanation. However, the point of view advocated in this paper strongly favors the physics proposed by Bilj et al as the essential explanation for the formation of thick superfluid ${ }^{4} \mathrm{He}$ films.

\section{TRANSFER PHENOMENA IN SUPERFLUIDS AND SUPERCONDUCTORS}

F. London wrote a paper in 1945 entitled "Planck's constant and low temperature transfer" [13]. The paper discusses in a unified way the low temperature transfer of mass in superfluid He films and in superconductors carrying a current near the surface. The same concepts were discussed in the work of Mendelssohn and of Daunt and Mendelssohn [11, 12]. London defines the mass transfer rate as

$$
R=\int j d x
$$

with

$$
j=m n v_{s}
$$

the mass current density for a superfluid with particles of mass $m$ and number density $n$ moving at average speed $v_{s}$ assumed parallel to the surface, and $x$ the direction perpendicular to the surface. $R$ gives the mass transferred per unit time along a surface of unit width. It has units $\mathrm{g} / \mathrm{cm} \cdot \mathrm{s}$, which is also angular momentum per unit volume, or angular momentum density. Hence $R / n$, with $n$ the superfluid number density, or the number density of superconducting electrons, has units of angular momentum. London remarks that the maximum value of $R / n$ observed experimentally ( $\equiv R_{c} / n$ ) both in superfluid ${ }^{4} \mathrm{He}$ and in superconductors, is of order $\hbar$, more precisely 26]:

$$
R_{c}=n \frac{\hbar}{2}
$$

where $n$ is the number density of the carriers giving rise to the mass transfer. For the superfluid flowing in a layer of thickness $d$, the transfer rate is

$$
R=n m v_{s} d
$$

(where the mass $m$ is $m_{e}$ for the superconductor, $m_{H e}$ for ${ }^{4} \mathrm{He}$ ), so that Eq. (15) yields

$$
\frac{R_{c}}{n}=m v_{c} d=\frac{\hbar}{2}
$$


where we denote by $v_{c}$ the critical velocity.

For ${ }^{4} \mathrm{He}$ we have

$$
m_{H e}=6.65 \times 10^{-24} \mathrm{~g}
$$

and the number density is

$$
n=2.182 \times 10^{22} \mathrm{~cm}^{-3} .
$$

The thickness of superfluid ${ }^{4} \mathrm{He}$ films is found to be approximately $300 \AA$. The condition Eq. (17) yields for the critical transfer speed

$$
v_{c}=26.4 \mathrm{~cm} / \mathrm{s}
$$

and for the critical mass transfer rate

$$
R_{c}=1.15 \times 10^{-5} \frac{\mathrm{g}}{\mathrm{cm} \cdot \mathrm{s}} .
$$

or for the critical volume transfer rate

$$
\frac{R_{c}}{\mathrm{~nm}}=0.793 \times 10^{-4} \frac{\mathrm{cm}^{3}}{\mathrm{~cm} \cdot \mathrm{sec}}
$$

These values are close to what is usually found experimentally [15, 16]. Mendelssohn and coworkers have shown in a series of detailed experiments that when different film flow rates are found it can be attributed to extraneous effects such as impurities adsorbed on the surfaces that change the effective geometrical transfer perimeter 31]. Daunt and Mendelssohn have furthermore shown [32] that the critical transfer rate is the same when the film flow is driven by heat supplied to the container (thermo-mechanical effect) as when it is driven by a gravitational potential difference.

For superconductors we have for the current density $J=n_{s} e v_{s}$, with $n_{s}$ the density of superconducting charge carriers, so that the mass transfer rate is

$$
R=\frac{m_{e}}{e} \int J d x=-\frac{m_{e} c}{4 \pi e} \int \frac{\partial B}{\partial x} d x=-\frac{m_{e} c}{4 \pi e} B
$$

where $B$ is the magnetic field and we have used $\vec{\nabla} \times \vec{B}=$ $\frac{4 \pi}{c} \vec{J}$. The magnetic field $\vec{B}$ points along the $+\hat{y}$ direction and varies in the $\hat{x}$ direction, the charge current flows in the $+\hat{z}$ direction and the mass current flows in the $-\hat{z}$ direction, and the surface is in the $y-z$ plane, as shown in Fig. 3. The critical transfer rate is then

$$
R_{c}=-\frac{m_{e} c}{4 \pi e} H_{c 1}
$$

where $H_{c 1}$ is the lower critical field given by Eq. (6):

$$
H_{c 1}=\frac{\hbar c}{4|e| \lambda_{L}^{2}}=\frac{\phi_{o}}{4 \pi \lambda_{L}^{2}}
$$

with $\phi_{0}=h c / 2 e$ the flux quantum. Eq. (24) yields a flux $\phi_{0}$ for magnetic field $H_{c 1}$ through a circle of radius $2 \lambda_{L}$. The critical transfer rate Eq. (23) is then

$$
R_{c}=\frac{m_{e} c^{2}}{4 \pi e^{2} \lambda_{L}^{2}} \frac{\hbar}{4} .
$$

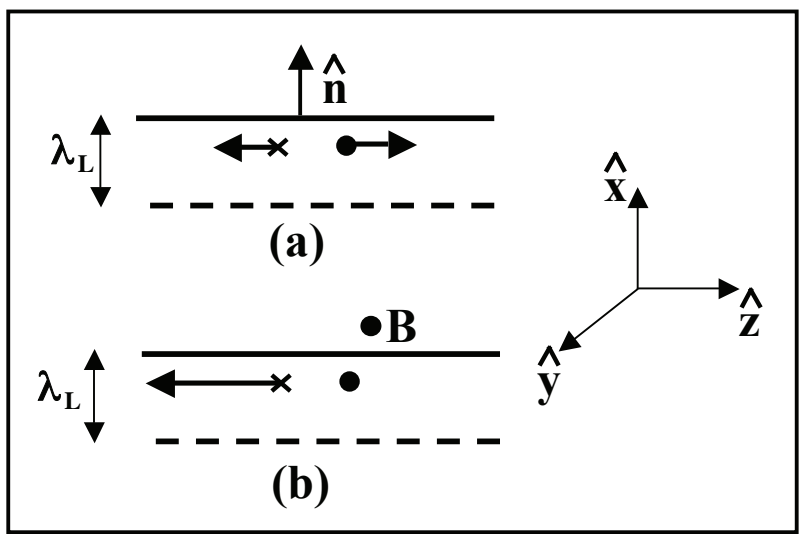

FIG. 3: The flow of carriers near the surface of a superconductor is shown. The normal to the surface points in the $\hat{x}$ direction. In (a) no magnetic field exists and no charge current flows. Electrons with spin pointing in the $+\hat{y}(-\hat{y})$ direction (out of and into the paper respectively) move in the $+\hat{z}(-\hat{z})$ direction with average speed $v_{\sigma}^{0}$ (Eq. (3)). In (b), a magnetic field $B$ of magnitude equal to the lower critical field $H_{c 1}$ exists pointing in the $\hat{y}$ direction. Electrons with spin pointing into the paper move at average speed $2 v_{\sigma}^{0}$ and those with opposite spin have stopped.

or, using Eq. (8)

$$
R_{c}=n_{s} \frac{\hbar}{4}=n_{\sigma} \frac{\hbar}{2}
$$

Here, $n_{\sigma}=n_{s} / 2$ is the density of carriers of one spin only. Eq. (26) agrees with London's formula Eq. (15) if the electrons contributing to the mass current are electrons of one spin orientation only.

This result follows naturally within the theory of hole superconductivity. In the absence of applied magnetic field, carriers of opposite spin move along the surface in opposite directions with speed given by $v_{\sigma}^{0}$ (Eq. (3)) within a layer of thickness $d=\lambda_{L}$ from the surface, as shown in Fig. 3(a). When the applied magnetic field reaches the value $H_{c 1}$, electrons of one spin orientation come to a stop and those of opposite spin orientation move at speed $2 v_{\sigma}^{0}$, as discussed in Sect. II. The critical transfer rate Eq. (15) is

$$
\frac{R_{c}}{n_{\sigma}}=m_{e}\left(2 v_{\sigma}^{0}\right) \lambda_{L}=m_{e}\left(2 \frac{\hbar}{4 m_{e} \lambda_{L}}\right) \lambda_{L}=\frac{\hbar}{2}
$$

in agreement with Eqs. (15) and (26). Thus, the theory of hole superconductivity allows for a simple interpretation of the London transfer equation Eq. (15).

\section{QUANTUM ZERO-POINT DIFFUSION}

In a superconducting wire fed by normal metal leads, the transport of current occurs with no drop in electric potential across the superconductor, i.e. in the absence 


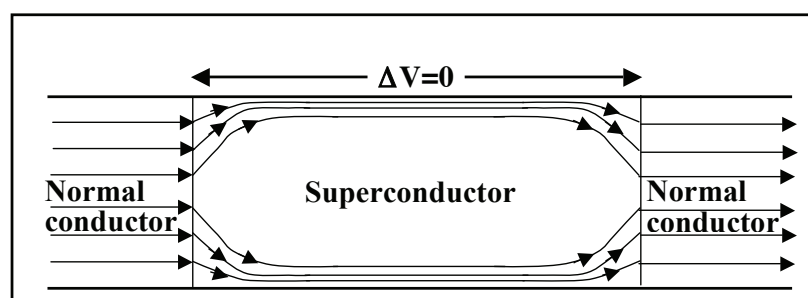

(a)

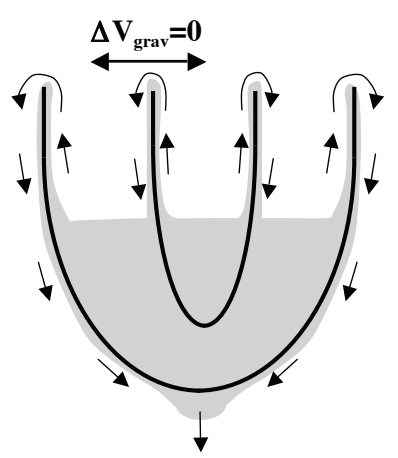

(b)
FIG. 4: (a) Current flow in a superconducting wire which is fed by normal conducting leads. The flow lines are calculated in Ref. 33]. There is no electric potential difference between both ends of the superconductor. (b) Flow of superfluid ${ }^{4} \mathrm{He}$ along surfaces in double beaker experiment. There is flow of superfluid from the inner to the outer beaker and from the outer beaker to the exterior, gradually emptying both beakers. However the levels in the inner and outer beaker are always identical throughout this process, so there is no gravitational potential difference between them $\left(\Delta V_{\text {grav }}=0\right)$.

of an accelerating force, as shown schematically in Fig. 4(a) . Daunt and Mendelssohn have constructed a very interesting analog of this situation for superfluid ${ }^{4} \mathrm{He}$, the "double beaker" experiment shown schematically in Fig. 4(b) 34]. Two concentric vessels are filled with liquid $\mathrm{He}$, and $\mathrm{He}$ will flow spontaneously from the inner vessel through the outer vessel to the outside. The key observation is that the levels of the inner and outer vessels remain identical throughout the process. Thus, there is no gravitational potential difference between the inner and outer vessels, hence no gravitational force driving the fluid from the inner to the outer vessel. The transfer of matter from the inner to the outer vessel without a driving force parallels the transport of current across the superconducting part of the wire in Fig. 4(a) without electric potential difference between its ends.

Mendelssohn has strongly advocated the view that "the momentum of frictionless transfer is derived from zeropoint energy" and that these transport phenomena in superconductors and superfluid ${ }^{4} \mathrm{He}$ can only be explained by "zero-point diffusion" [11]. He denotes the superfluid particles as "z-particles", and postulates that these transfer processes occur because the z-particles have zeropoint energy, and that the transport processes in the superconductor and liquid helium films "is simply due to the diffusion of z-particles under their zero-point momentum". In his words, "If at some place outside (dx), $z$-particles are removed, zero-point diffusion must take place and a macroscopic flow of z-particles in the $x$ direction will occur. This process entails no actual acceleration of z-particles but merely a greater mean free path in the x-direction."

Mendelssohn emphasizes that the Bose-Einstein gas has no zero-point energy, and therefore it cannot provide an explanation for this pressure-independent flow. He states "The process of zero-point diffusion which we have introduced in order to explain these phenomena does not exist in the ideal Bose-Einstein gas." Therefore, Mendelssohn concludes "According to our considerations, zero-point energy is necessary for the appearance of frictionless transport, and in fact no condensation to zero velocity takes place in superconductors and probably not in liquid helium either."

In other words, the conventional understanding of superfluidity as described by Bose condensation into a macroscopic $p=0$ state cannot explain the film flow in superfluid $\mathrm{He}$, and the conventional understanding of superconductivity as a condensate into the conventional BCS state cannot explain the current flow in superconductors. We fully agree.

Instead, the ground state of superconductors proposed within the theory of hole superconductivity does possess macroscopic zero-point motion, as shown schematically in Fig. 5(a). The "zero-point diffusion" in this case however does result in a change in the speed of the individual electrons when a charge current flows (Fig. 5(b)). For example, for electrons near the upper surface those with spin pointing into (out of) the paper speed up (slow down). The force responsible for these changes is simply the transient Faraday emf associated with the changing magnetic field in going from Fig. 5(a) to Fig. 5(b). Nevertheless, confirming Mendelssohn's intuition, the critical speed associated with the critical transfer rate is found to be closely related to the speed of the pre-existent zeropoint motion Eq. (3).

Thus, following Mendelssohn's reasoning, the analogous behavior of $\mathrm{He}$ film flow and superconducting charge flow leads us to conclude that superfluid ${ }^{4} \mathrm{He}$ must possess ground state zero-point motion closely related to the zero-point motion predicted in superconductors within the theory of hole superconductivity.

Conventional quantum mechanics predicts zero-point momentum $p \sim \hbar / d$ perpendicular to the surface for a film of thickness $d$ on a surface. However it does not predict that this momentum will redirect itself in direction parallel to the surface. Instead, in the $2 \lambda_{L}$ orbits predicted within our model, the magnitude of the zero-point velocity can be understood with conventional quantum mechanics as arising from confinement in a surface layer of order of the London penetration depth and oriented perpendicular to the surface, yet the resulting motion of the spin current is in direction parallel to the surface 23 . 


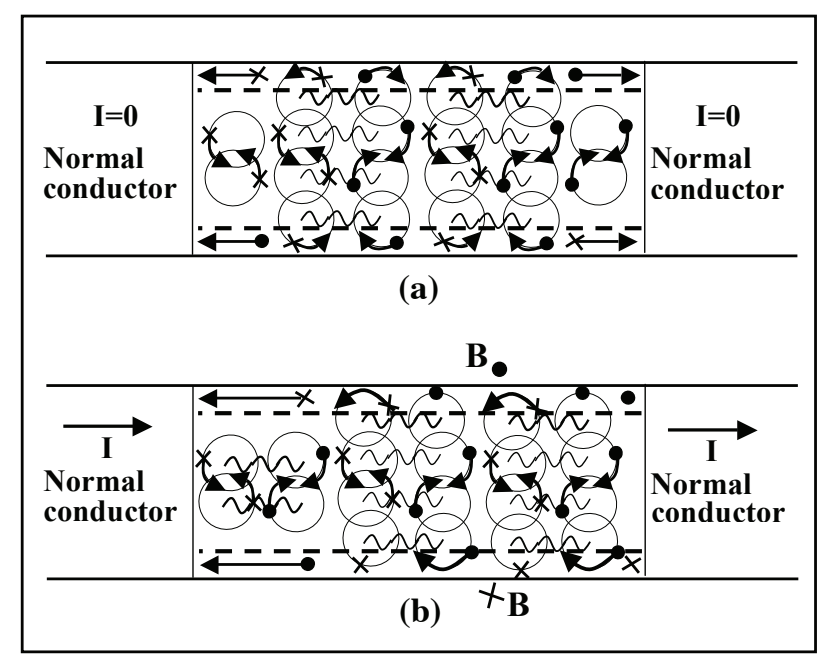

FIG. 5: Superconducting wire, some $2 \lambda_{L}$ orbits are shown schematically. The wiggly lines connect Cooper pair partners. (a) In the absence of a charge current, a pure spin current circulates. Near the upper surface, electrons with spin into (out of) the paper circulate to the left (right), near the lower surface the situation is reversed. There are also vertical spin currents near the vertical boundaries of the superconductor (not shown). In the interior of the superconductor the spin currents cancel out. (b) In the presence of a charge current, electrons of spin into the paper move faster near the upper surface (where the magnetic field points out of the paper), and electrons of spin out of the paper move faster near the lower surface (where the magnetic field points into the paper). There is a net charge current to the right and a net mass current to the left.

This change of direction is closely associated with the fact that this zero-point motion is rotational. We conclude therefore that ${ }^{4} \mathrm{He}$ must also possess rotational zero point motion.

\section{QUANTUM PRESSURE, UNCERTAINTY, AND ROTATIONAL ZERO POINT MOTION}

In conventional quantum mechanics there is no "rotational zero-point motion". For example, a quantum particle in a ring, whether a fermion or a boson, has a ground state orbital wave function which is constant as function of the azimuthal angle and has orbital angular momentum $L=0$ according to the Schrodinger equation.

Instead, we have proposed [35, 36] that electrons in rings have minimum angular momentum $\hbar / 2$, and hence rotational zero-point energy (just like in superconductors according to the theory of hole superconductivity) originating in an intrinsic double-valuedness of the electron's orbital wavefunction.

The assumption that electrons have rotational zeropoint motion provides a new understanding of electronic "quantum pressure" [4, 28]. The kinetic energy of a particle of mass $m$ and orbital angular momentum $L$ in an

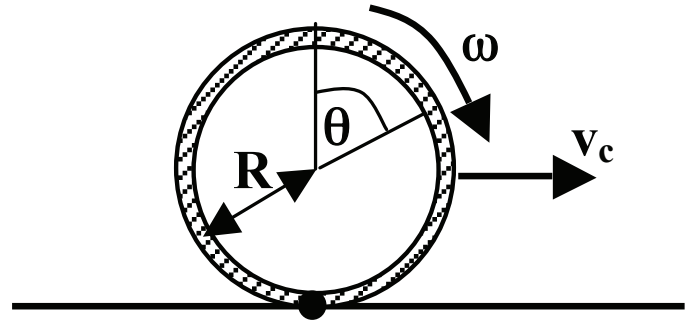

FIG. 6: Mechanical picture of a quantum-mechanical particle propagating with speed $v_{c}$. It has a "radius" $R=\hbar / m_{e} v_{c}$, circumference $\lambda=2 \pi R=h / m_{e} v_{c}$, rolls without slipping with angular frequency $\omega=v_{c} / R$, has linear momentum $p=h / \lambda$, kinetic energy $E=\hbar \omega$ and angular momentum $L=\hbar$.

orbit of radius $R$ is $L^{2} / 2 m R^{2}$, of the same form as the kinetic energy in quantum mechanics for $L \sim \hbar$. For $L$ non-zero, reducing the region that the particle occupies would decrease $R$ and increase the kinetic energy and thus provides a mechanical explanation of "quantum pressure". Similarly we can understand Heisenberg's uncertainty principle $\Delta p \Delta x \sim \hbar$ "mechanically", if the left side actually represents an angular momentum of the particle.

Since a boson exerts "quantum pressure" just like a fermion does and is also subject to Heisenberg's uncertainty principle, we are led to the conclusion that bosons too must possess rotational zero-point motion, contrary to the predictions of Schrodinger's equation. We believe that this is strikingly confirmed by the properties of ${ }^{4} \mathrm{He}$ films discussed in this paper, that vividly display this motion. More generally, also other peculiar transport properties of ${ }^{4} \mathrm{He}$ according to Mendelssohn [39] are clear evidence that the superfluid exerts "zero-point pressure" that is not accounted for in the physics of Bose condensation; they would be accounted for by quantum pressure resulting from zero-point rotation, just as we have proposed for superconductors.

If "quantum pressure" and Heisenberg's uncertainty principle are associated with rotational motion, it is natural to conclude that the quantum "phase" of a particle's wavefunction is associated with an azimuthal angle of rotation. So let us picture a quantum particle of mass $m$ as a "rim" of radius R, and assume that "phase coherent" propagation corresponds to rolling without slipping as depicted in Fig. 6. If the center of mass is moving with speed $v_{c}$, the angular velocity for rolling without slipping is

$$
\omega=v_{c} / R
$$

and the angular momentum is

$$
L=I \omega=m R^{2} \omega
$$

with $I=m R^{2}$ the moment of inertia. The linear mo- 


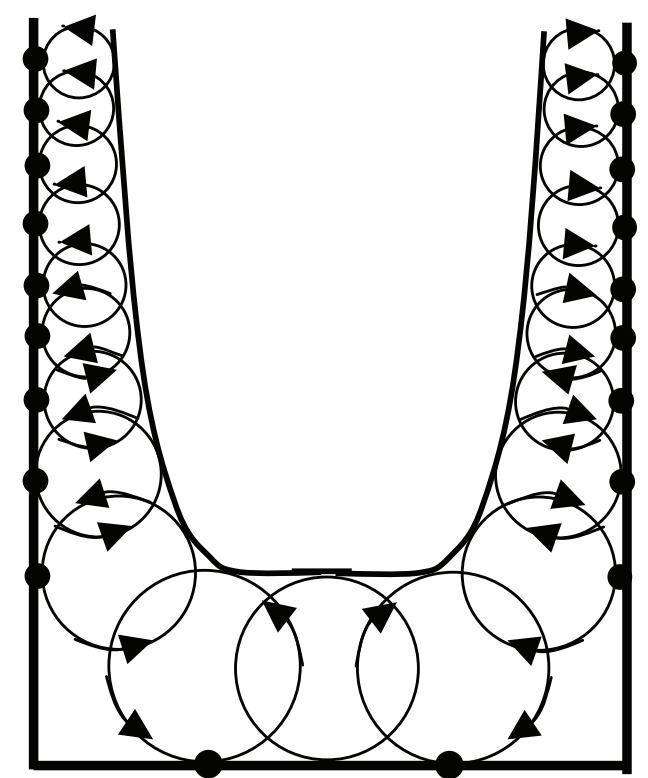

FIG. 7: Schematic depiction of ${ }^{4} \mathrm{He}$ in the superfluid phase. The atoms undergo rotational zero-point motion with radius as large as possible compatible with the geometrical constraints. The film flow against gravity results from rolling without slipping of the atomic orbits along the vertical surfaces of the container, driven by the rotational zero-point motion.

mentum is given by

$$
p=m v_{c}=\frac{L}{R}=\frac{h}{\lambda}
$$

if we assume $\lambda=2 \pi R$, i.e. that the circumference of the rim is it's "wavelength", and $L=\hbar$ is its angular momentum. The kinetic energy is the sum of translational and rotational kinetic energies:

$$
E=\frac{1}{2} m v_{c}^{2}+\frac{L^{2}}{2 I}=m \omega^{2} R^{2}=L \omega=\hbar \omega=h f
$$

with $f=\omega / 2 \pi$ the frequency of rotation.

Thus, we obtain the well-known de Broglie relations Eqs. (30) and (31), consistent with the laws of mechanics, without assuming that the particle is a "wave" nor the existence of a "wave packet" (necessary to account for the factor of 2 difference between group velocity and phase velocity in the conventional theory). The radius $R$ is given by $R=\hbar / m_{e} v_{c}$ for a particle propagating with speed $v_{c}$. If the particle is confined to a region $\Delta x$ this will force $R$ to be smaller than $\Delta x$ and the frequency of rotation $\omega=\hbar / m R^{2}$ will increase, thus increasing the kinetic energy $\hbar \omega$.

In ${ }^{4} \mathrm{He}$, the atoms condense into a coherent wave function describing all the atoms in the condensate. We assume that just as in the description of superconductors within the theory of hole superconductivity, the
${ }^{4} \mathrm{He}$ atoms will undergo macroscopic rotational zeropoint motion coherently, as shown schematically in Fig. 7. Unlike the case of superconductors where there is the length $\lambda_{L}$ determined by the mass and charge of the electron and the density (Eq. (8)), the length scale of rotational zero-point motion here is presumably determined by the geometrical constraints and grows as large as possible to minimize the zero-point kinetic energy, as shown in Fig. 7. The "rolling without slipping" motion of the He atom "rims" causes the liquid to climb up the walls of the container, driven by the energy of zero-point motion. The thickness of the films coating the surface as function of height can be understood using the model of Bilj et al [14].

Other proposals that revise fundamental aspects of quantum mechanics have been made recently. Nikulov [37] postulates the existence of an azimuthal "quantum force" to explain the generation of currents in superconducting rings. In contrast to what is discussed here, Nikulov's force would only act when phase coherence is established. Hestenes 38 in a series of papers has proposed a rotational "zitterbewegung" for electrons that would manifest itself "in every application of quantum mechanics". These proposals may be related to the physics discussed here.

\section{DISCUSSION}

The theory of hole superconductivity predicts that (1) kinetic energy lowering plays a key role in the physics of superconductivity, since superconductivity is driven by kinetic energy lowering; (2) the wavefunction of the superfluid electrons expands in the transition to superconductivity; (3) negative charge is expelled from the interior of the superconductor to the surface and beyond; (4) macroscopic zero-point motion exists, in the form of a spin current, in the ground state of superconductors; (5) the 'Meissner pressure' 33] that allows superconductors to expel magnetic fields in defiance of Faraday's electromotive force is quantum pressure originating in kinetic energy lowering. None of these predictions is part of the conventional understanding of superconductivity within London-BCS theory, and none of these predictions has yet been experimentally verified.

So it is indeed remarkable that each of these predictions has an already known counterpart in the physics of superfluid ${ }^{4} \mathrm{He}$, namely (following the same numbering as in the previous paragraph): (1) kinetic energy lowering causes ${ }^{4} \mathrm{He}$ to expand its volume, from what it would be to optimize the Van der Waals potential energy, to almost 3 times larger, and causes ${ }^{4} \mathrm{He}$ to remain liquid under its own vapor pressure down to zero temperature; (2) in the transition to superfluidity, ${ }^{4} \mathrm{He}$ expands further as the temperature is lowered below the $\lambda$ point (negative thermal expansion coefficient below $T_{\lambda}$ ); (3) mass is expelled from the interior of a ${ }^{4} \mathrm{He}$ container to the exterior (Onnes effect); (4) macroscopic motion of the superfluid 
occurs spontaneously in Rollin films; (5) the Rollin film spontaneously climbs the wall of the container, in defiance of the force of gravity.

This coincidence suggests that (i) superfluidity and superconductivity are even more closely related than conventionally believed, and (ii) that the predictions of the theory of hole superconductivity for superconductors are likely to be true. In contrast, in the conventional understanding, while many analogies in the behavior of superconductors and superfluid ${ }^{4} \mathrm{He}$ are clearly recognized [18], kinetic energy lowering plays absolutely no role in superconductivity. And while several other proposals have been made for kinetic-energy lowering mechanisms to explain 'unconventional' superconductivity in the high $T_{c}$ cuprates 40 56], no connection between any of these mechanisms and the physics of superfluid ${ }^{4} \mathrm{He}$ has been proposed so far to our knowledge.

The fact that superfluid ${ }^{4} \mathrm{He}$, a macroscopic quantum system, will spontaneously climb the walls of a container, vividly suggests that one is witnessing a macroscopic manifestation of quantum zero-point motion, i.e. the inability of quantum particles to remain at rest. The fact that the transfer rate per unit density $(R / n)$ is of order $\hbar$ lends strong support to this conception. It is really remarkable that in the generally accepted understanding of ${ }^{4} \mathrm{He}$ quantum zero point motion plays absolutely no role in the creeping up of helium along walls 15 18]. This is because the conventional understanding of ${ }^{4} \mathrm{He}$ as a Bose condensate does not provide an explanation for it: while confinement over a film thickness $d$ would raise the zero-point momentum in direction perpendicular to the surface, it would not give rise to zero-point momentum in direction parallel to the surface.

The properties of superconductors listed in the first paragraph result from the prediction that superconducting electrons undergo rotational zero-point motion[5]. Thus, we are led to the prediction that superfluid ${ }^{4} \mathrm{He}$ atoms also undergo rotational zero-point motion. The fact that the mass transfer rate per unit number density is of order $\hbar$ for both superconducting current and superfluid $\mathrm{He}$ films, observed long ago but not understood, has a simple explanation within the framework presented here: the critical speed is the speed of macroscopic ground state rotational zero-point motion in both superconductors and superfluid ${ }^{4} \mathrm{He}$.

This prediction has not yet been verified either for superconductors or superfluid ${ }^{4} \mathrm{He}$. However it is well known that rotational motion plays a fundamental role both in superconductors (e.g. vortices in type II materials) and in superfluid ${ }^{4} \mathrm{He}$ (vortices, "rotons"). The point of view discussed in this paper suggests that rotational motion in both systems pre-exists in the ground state as zero-point motion, and is merely made more apparent in excited states.

Beyond superfluids and superconductors, we have proposed that rotational zero-point motion would provide an alternative and more compelling explanation of quantum pressure, Heisenberg's uncertainty principle, and the stability of matter, than the conventional explanation based on the Schrodinger equation[4]. We have shown here that rotating particles rolling without slipping propagate according to de Broglie's relations. For electrons, we have proposed that rotational zero-point motion arises from an intrinsic double-valuedness of the orbital wavefunction [35, 36], intimately tied to the electron's half-integer spin. However, the considerations in this paper lead to the conclusion that a system of ${ }^{4} \mathrm{He}$ atoms, bosons, also undergoes rotational zero-point motion. If so, the fundamental origin of rotational zeropoint motion cannot be attributed to half-integer spin. On the other hand it is satisfying that "quantum pressure" would have a unified explanation for both fermions and bosons as arising from rotational zero-point motion, as is the fact that the fundamental constant $\hbar$ that determines the behavior of both fermions and bosons in quantum mechanics, has units of angular momentum.

Thus, the considerations in this paper suggest that Schrodinger's equation, as well as Dirac's equation and Klein Gordon's equation from which Schrodinger's equation derives for fermions and bosons respectively, are inadequate to provide a general description of reality, because they do not predict rotational zero-point motion. If so, the correct equations remain to be discovered.
[1] See for example C. J. Gorter, Progr. Low Temp. Phys. I, 1 (1955) and references therein.

[2] F. London has written a two-volume book series entitled 'Superfluids', where Volume I deals with superconductors and Volume II with superfluid ${ }^{4} \mathrm{He}$, emphasizing the common aspects of the phenomena both through the title of the series and throughout its contents.

[3] K. Mendelssohn, Physics Today, April 1969, p. 47, and references therein.

[4] J.E. Hirsch, Int. J. Mod. Phys. 25, 1173 (2011) and references therein.

[5] J.E. Hirsch, Europhys. Lett. 81, 67003 (2008).

[6] J.E. Hirsch, Phys. Lett. A 309, 457 (2003).

[7] H. Kamerlingh Onnes, Leiden Comm. 159, Trans. Fara- day Soc. 18, No. 53 (1922).

[8] B.V. Rollin and F. Simon, Physica 6, 219 (1939).

[9] J.G. Daunt and K. Mendelssohn, Nature 141, 911 (1938); 142, 475 (1938); 143, 719 (1939); 157, 839 (1946); Proc. Roy. Soc. London A170, 423 (1939).

[10] R.P. Feynman, Progr. Low Temp. Phys. Vol. I, p. 17 (1955).

[11] K. Mendelssohn, Proc. Phys. Soc. London 57, 371 (1945).

[12] J.G. Daunt and K. Mendelssohn, Nature 150, 604 (1942); Phys. Rev. 69, 126 (1946).

[13] F. London, Rev. Mod. Phys. 17, 310 (1945).

[14] A. Bilj, J. De Boer and A. Michels, Physica 8, 655 (1941).

[15] K.R. Atkins, "Liquid Helium", Cambridge Universtiy Press, Cambridge, 1959. 
[16] J. Wilks, "Liquid and solid helium", Clarendon Press, Oxford, 1967.

[17] D. F. Brewer, in "The physics of liquid and solid helium", ed. by K. H. Bennemann and J. B. Ketterson, Wiley, New York, 1978, p. 573.

[18] D.R. Tilley and J. Tilley, "Superfluidity and Superconductivity", Institute of Physics Publishing, Bristol, 1990.

[19] M. Tinkham, "Introduction to Superconductivity", 2nd ed., McGraw-Hill, New York, 1996.

[20] J.E. Hirsch, Physica C 470, 635 (2010).

[21] D.N. Basov et al, Science 283, 49 (1999); H. J. A. Molegraaf et al, Science 295, 2239 (2002); A. F. SantanderSyro et al, Europhys. Lett., 62568 (2003); A. Charnukha et al, Nature Communications 2, 219 (2011).

[22] J.E. Hirsch, arXiv:1106.5311 (2011).

[23] J.E. Hirsch, Ann. der Physik 17, 380 (2008).

[24] J.E. Hirsch, J. Phys. Cond. Matt. 20, 235233 (2008).

[25] F. Simon, Nature 133, 529 (1934); K. Bennewitz and F. Simon, Z. fur Physik 16, 183 (1923).

[26] F. London, Superfluids, Vol. II, John Wiley and Sons, Hoboken, New Jersey, 1954.

[27] K.R. Atkins and M.H. Edwards, Phys. Rev. 97, 1429 (1955).

[28] J.E. Hirsch, Jour. of Superconductivity and Novel Magnetism 23, 309 (2010).

[29] K.R. Atkins, in ref. [15], p. 226.

[30] J.E. Hirsch, Phys. Rev. 59, 436 (1999) and references therein.

[31] J.B. Brown and K. Mendelssohn, Proc. Phys. Soc. A63, 1312 (1950); R. Bowers and K. Mendelssohn, Proc. Phys. Soc. A63, 1318 (1950); K. Mendelssohn and G.K. White, Proc. Phys. Soc. A63, 1328 (1950).

[32] J.G. Daunt and K. Mendelssohn, Proc. Phys. Soc. A63, 1305 (1950).

[33] F. London, Superfluids, Vol. I, Dover Publications, New York, 1961.

[34] J.G. Daunt and K. Mendelssohn, Nature 157, 839 (1946).

[35] J.E. Hirsch, Mod. Phys. Lett. B 24, 2201 (2010).

[36] J.E. Hirsch, Phys. Lett. A 374, 3777 (2010).

[37] A.V. Nikulov, Phys.Rev. B 64, 012505 (2001); arXiv:1104.4856 (2011).

[38] D. Hestenes, Found. of Phys. 20, 1213 (1990); Found. of
Phys. 40, 1 (2010).

[39] K. Mendelssohn, "Report of an International Conference on Fundamental Particles and Low Temperatures", Physical Society of London, 1947, Vol. II, p. 35.

[40] V. J. Emery and S. A. Kivelson, Jour. Phys. Chem. Solids 61, 467 (2000).

[41] S. Chakravarty, Hae-Young Kee and E. Abrahams, Phys. Rev. B 67, 100504 (2003).

[42] E. W. Carlson, V. J. Emery, S. A. Kivelson and D. Orgad, in "Superconductivity: Conventional and Unconventional Superconductors", ed. by K. H. Bennemann and John B. Ketterson, Springer, Berlin, 2008, p. 12251348 .

[43] M. R. Norman, M. Randeria, B. Jank and J. C. Campuzano, Phys. Rev. B 61, 14742 (2000).

[44] P. Wrobel, R. Eder and R. Micnas, J. Phys. Condens. Matter 15, 2755 (2003)

[45] T. Eckl, W. Hanke and E. Arrigoni, Phys. Rev. B 68, 014505 (2003).

[46] R. Haslinger and A. V. Chubukov, Phys. Rev. B 68, 214508 (2003).

[47] S. Feng, Phys. Rev. B 68, 184501 (2003).

[48] M. Ogata, H.Yokoyama, Y. Yanase, Y. Tanaka and H.Tsuchiura, Jour. Phys. Chem. Solids 67, 37 (2006) .

[49] Th. A. Maier, M. Jarrell, A. Macridin and C. Slezak, Phys. Rev. Lett. 92, 027005 (2004).

[50] Z. C. Gu, Tao Li, and Z. Y. Weng, Phys. Rev. B 71, 064502 (2005).

[51] B. Kyung, A. Georges and A.-M. S. Tremblay, Phys. Rev. B 74, 024501 (2006).

[52] J.P. Carbotte and E. Schachinger, J. Low Temp. Phys. 144, 61 (2006).

[53] K. Haule and G. Kotliar, Europhysics Letters 77, 27007 (2007).

[54] M. R. Norman, A. V. Chubukov, E. van Heumen, A. B. Kuzmenko, and D. van der Marel, Phys. Rev. B 76, 220509(R) (2007)

[55] Saurabh Maiti and Andrey V. Chubukov, Phys. Rev. B 81, 245111 (2010)

[56] L. Vidmar and J. Bonca, Phys. Rev. B 82, 125121 (2010). 